\title{
Stability of Two Superposed Elasticoviscous Liquids in Plane Couette Flow
}

\author{
CHin-Hsiu LI \\ Department of Engineering Mechanics, The University of Michigan \\ Ann Arbor, Michigan \\ (Received 5 September 1968; final manuscript received 26 November 1968)
}

\begin{abstract}
It is found that the elasticity in the liquid cannot only destabilize the flow, but it can also stabilize the flow for certain values of depth ratio, viscosity ratio, and elasticity ratio. The stabilizing or destabilizing effect of the elasticity in liquid is absent in the absence of viscosity stratification, and is only brought about when the viscosity varies.
\end{abstract}

\section{INTRODUCTION}

Oldroyd ${ }^{1}$ proposed a mathematical model to describe the mechanical behavior of viscoelastic material. This model has proved very useful in characterizing the rheological properties of certain dilute polymer solutions at sufficiently small rates of strain. Using this model, Thomas and Walters, ${ }^{2,3}$ Herbert," and $\mathrm{Lai}^{\mathrm{b}}$ examined the stability of specific cases of flow. Recently, Chun and Schwarz ${ }^{6}$ adopted Coleman and Noll's ${ }^{7}$ model of a second-order fluid and considered the effect of slight viscoelasticity on the hydrodynamic stability of a plane Poiseuille flow. They all showed that the elastic behavior in elasticoviscous liquid destabilizes the flows. $\mathrm{Yih}^{8}$ considered plane Couette-Poiseuille flow of two superimposed layers of Newtonian fluids of different viscosities. It was found that the viscosity stratification destabilizes the flow in some ranges of the depth and viscosity ratios, and stabilizes the flow in others.

Investigation of the plane Couette flow of two superimposed layers of elasticoviscous liquid of different viscosities and different elasticities is made. The prototype of liquid designed by Oldroyd ${ }^{1}$ is considered. For this liquid, the equations of state are

$$
S_{i k}=-p g_{i k}+p_{i k},
$$

and

$$
\left(1+\lambda_{1} \frac{d_{c}}{d t}\right) p^{i k}=2 \eta_{0}\left(1+\lambda_{2} \frac{d_{c}}{d t}\right) \epsilon^{i k}
$$

${ }^{1}$ J. G. Oldroyd, Proc. Roy. Soc. (London) A245, 278 (1958).

2 R. H. Thomas and K. Walters, Proc. Roy. Soc. (London) A274, 371 (1963).

R. H. Thomas and K. Walters, J. Fluid Mech. 18, 33 (1964).

D. M. Herbert, J. Fluid Mech. 17, 353 (1963).

5 W. Lai, Phys. Fluids 10, 844 (1967).

D D. H. Chun and W. H. Schwarz, Phys. Fluids 11, 5 (1968).

7 B. D. Coleman and W. Noll, Arch. Ratl. Mech. Anal. $6,355(1960)$.

${ }^{8}$ C.-S. Yih, J. Fluid Mech. 27, 337 (1967). in which $S_{i k}$ is the stress tensor, $p$ an arbitrary isotropic pressure, $g_{i k}$ the metric tensor of a fixed coordinate system, $\epsilon_{i k}=\frac{1}{2}\left(u_{i, k}+u_{k, i}\right)$ the rate-ofstrain tensor, $\eta_{0}$ a coefficient of viscosity, $\lambda_{1}$ the relaxation time, and $\lambda_{2}$ the retardation time. The coeffcients $\eta_{0}, \lambda_{1}$, and $\lambda_{2}\left(<\lambda_{1}\right)$ are all positive. The symbol $d_{c} / d t$ denotes the convective derivative of a tensor quantity in relation to the fluid in motion. For a contravariant tensor $T^{i j}$

$$
\left(\frac{d_{c}}{d t}\right) T^{i i}=\left(\frac{\partial}{\partial t}\right) T^{i j}+T_{, m}^{i i} V^{m}-T^{m i} V_{, m}^{i}-T^{i m} V_{, m}^{j} .
$$

It is found that the elasticity in the liquid cannot only destabilize the flow, but it can also stabilize the flow for certain values of depth ratio, viscous ratio, and elasticity ratio.

\section{THE PRIMARY FLOW}

Consider two elasticoviscous liquids I and II between two parallel walls having the upper boundary moving with a constant velocity $U_{0}$, the lower boundary being stationary (see Fig. 1). Let $\left[\left(u_{1}\right)_{k}\right.$, $\left.\left(u_{2}\right)_{k},\left(u_{3}\right)_{k}\right]$ denote the velocity components in the $x_{1}, x_{2}$, and $x_{3}$ directions, respectively, where $x_{1}, x_{2}$, and $x_{3}$ are Cartesian coordinates, $\left(p_{i i}\right)_{k}$ denotes the $i-j$ component of stress tensor, and $\left(\lambda_{1}\right)_{k}$ and $\left(\lambda_{2}\right)_{k}$ denote the relaxation time and retardation time. The

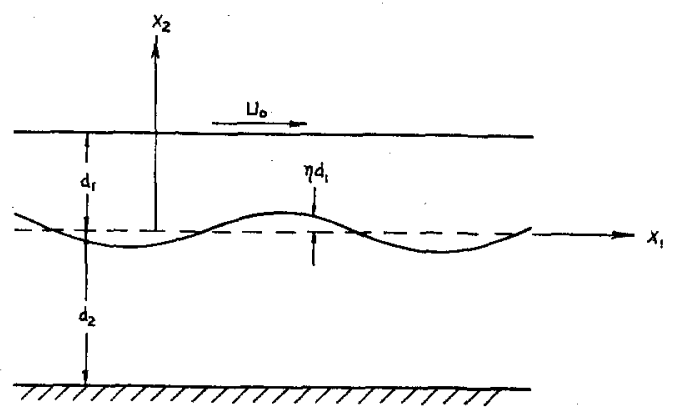

FIg. 1. Definition sketch. 
subscript $k$ is I for the upper liquid and II for the lower liquid since the equations of state and equations of motion governing liquid I and liquid II will be the same in the form. At this stage we drop the subscript for convenience, and consider the steady undisturbed flow

$$
u_{1}=\bar{u}\left(x_{2}\right), \quad u_{2}=0, \quad \text { and } \quad u_{3}=0 .
$$

For this flow, the constitutive equations can be written as follows:

$\bar{p}_{11}+\lambda_{1}\left(\frac{\partial \bar{p}_{11}}{\partial t}+\frac{\partial \bar{p}_{11}}{\partial x_{1}} \bar{u}-2 \bar{p}_{11} \frac{\partial \bar{u}}{\partial x_{2}}\right)=-2 \eta_{0} \lambda_{2}\left(\frac{\partial \bar{u}}{\partial x_{2}}\right)^{2}$,

$\bar{p}_{12}+\lambda_{1}\left(\frac{\partial \bar{p}_{12}}{\partial x_{1}} \bar{u}-\bar{p}_{22} \frac{\partial \bar{u}}{\partial x_{2}}\right)=\eta_{0} \frac{\partial u}{\partial x_{2}}$,

$\bar{p}_{13}+\lambda_{1}\left(\frac{\partial \bar{p}_{13}}{\partial x_{1}} \bar{u}-\bar{p}_{23} \frac{\partial u}{\partial x_{2}}\right)=0$,

$\bar{p}_{22}+\lambda_{1} \frac{\partial \bar{p}_{22}}{\partial x_{1}} \bar{u}=0$

$\bar{p}_{28}+\lambda_{1} \frac{\partial \bar{p}_{23}}{\partial x_{1}} \bar{u}=0$

$\bar{p}_{33}+\lambda_{1} \frac{\partial \bar{p}_{33}}{\partial x_{1}} \bar{u}=0$.

The equations of motion can be written as

$$
\begin{aligned}
& 0=-\frac{\partial \bar{p}}{\partial x_{1}}+\frac{\partial \bar{p}_{11}}{\partial x_{1}}+\frac{\partial \bar{p}_{21}}{\partial x_{2}}+\frac{\partial \bar{p}_{31}}{\partial x_{3}}, \\
& 0=-\frac{\partial \bar{p}}{\partial x_{2}}+\frac{\partial \bar{p}_{12}}{\partial x_{1}}+\frac{\partial \bar{p}_{22}}{\partial x_{2}}+\frac{\partial \bar{p}_{32}}{\partial x_{3}}+\rho g, \\
& 0=-\frac{\partial \bar{p}}{\partial x_{3}}+\frac{\partial \bar{p}_{13}}{\partial x_{1}}+\frac{\partial \bar{p}_{23}}{\partial x_{2}}+\frac{\partial \bar{p}_{33}}{\partial x_{3}}
\end{aligned}
$$

Equations (4) and (5) admit the stress components of primary flow to be

$$
\begin{aligned}
\bar{p}_{13} & =0, \quad \bar{p}_{22}=0, \quad \bar{p}_{23}=0, \quad \bar{p}_{33}=0, \\
\bar{p} & =\bar{p}\left(x_{2}\right), \quad \bar{p}_{11}=\bar{p}_{11}\left(x_{2}\right), \quad \text { and } \quad \bar{p}_{12}=\bar{p}_{12}\left(x_{2}\right) .
\end{aligned}
$$

We make all quantities nondimensional by letting

$$
\begin{gathered}
x_{1}=x d_{1}, \quad y_{1}=y d_{1}, \quad \bar{u}=U U_{0}, \quad \bar{p}=\rho_{\mathrm{I}} U_{0}^{2} P, \\
\bar{p}_{i j}=\rho_{\mathrm{I}} U_{0}^{2} P_{i j}, \quad \text { and } t=\frac{d_{1}}{U_{0}} \tau .
\end{gathered}
$$

The nondimensional forms of the first two equations in (4) and (5) are then

$$
\begin{gathered}
\left(P_{11}\right)_{i}-2\left(M_{1}\right)_{i}\left(P_{12}\right)_{i} \frac{d U_{i}}{d y}=-2 \beta_{i} \frac{\left(M_{2}\right)_{i}}{R}\left(\frac{d U_{i}}{d y}\right)^{2}, \\
\left(P_{12}\right)_{i}=\beta_{i} \frac{1}{R}\left(\frac{d U_{i}}{d y}\right)
\end{gathered}
$$

$$
\begin{gathered}
0=\frac{d}{d y}\left(P_{21}\right)_{i}, \\
0=-\frac{d}{d y} P_{i}-\delta_{i} F^{-2},
\end{gathered}
$$

in which $R=\left[U_{0} \rho_{\mathrm{I}} d_{1} /\left(\eta_{0}\right)_{\mathrm{I}}\right]$ is the Reynolds number, $\left(M_{1}\right)_{i}=\left[U_{0}\left(\lambda_{1}\right)_{i} / d_{1}\right],\left(M_{2}\right)_{i}=\left[U_{0}\left(\lambda_{2}\right)_{i} / d_{1}\right]$, and $F^{2}=U_{0}^{2} / g d_{1}$ is the Froude number. The subscript $i$ is taken to be I and II for the upper and the lower layer of liquids, respectively,

$$
\beta_{\mathrm{I}}=1, \quad \beta_{\mathrm{II}}=m_{\eta}, \quad m_{\eta}=\frac{\left(\eta_{0}\right)_{\mathrm{II}}}{\left(\eta_{0}\right)_{\mathrm{I}}}
$$

is the ratio of viscosity, and

$$
\delta_{\mathrm{I}}=1, \quad \delta_{\mathrm{II}}=\gamma, \quad \gamma=\frac{\rho_{\mathrm{II}}}{\rho_{\mathrm{I}}}
$$

is the ratio of density. From Eqs. (7b) and (7c) the equations governing the primary flow can be obtained. These are

$$
\frac{d^{2} U_{\mathrm{I}}}{d y^{2}}=0 \text { and } \frac{d^{2} U_{\mathrm{II}}}{d y^{2}}=0 .
$$

Subject to the boundary conditions that $U_{\mathrm{I}}$ is equal to a specified $U_{0}$ on the upper boundary and $U_{\text {II }}$ is zero on the lower boundary, and that $U_{\mathrm{I}}$ and $U_{\mathrm{II}}$, and $\left(P_{12}\right)_{\mathrm{I}}$ and $\left(P_{12}\right)_{\mathrm{II}}$ must be continuous at the interface, Eqs. (10) can be solved to yield the solutions

$$
U_{\mathrm{I}}=a_{1} y+b \text { and } U_{\mathrm{II}}=a_{2} y+b,
$$

in which

$$
\begin{array}{ll}
a_{1}=\frac{m_{\eta}}{m_{\eta}+n}, & b=\frac{n}{m_{\eta}+n}, \\
a_{2}=\frac{1}{m_{\eta}+n}, & n=\frac{d_{2}}{d_{1}} .
\end{array}
$$

The primary flow has the same velocity profile as that in the Newtonian fluid. As soon as we have $U_{1}$ and $U_{\mathrm{II}}$, from Eqs. $(7 \mathrm{a}-\mathrm{d})$ we obtain

$$
\begin{gathered}
\left(P_{12}\right)_{\mathrm{I}}=a_{1} / R, \quad\left(P_{11}\right)_{\mathrm{I}}=(2 / R)\left[\left(M_{1}\right)_{\mathrm{I}}-\left(M_{\mathrm{g}}\right)_{\mathrm{I}}\right] a_{1}^{2}, \\
\frac{d P_{\mathrm{I}}}{d y}=-F^{-2}, \quad\left(P_{12}\right)_{\mathrm{II}}=m_{\eta} \frac{a_{2}}{R}=\left(P_{12}\right)_{\mathrm{I}}, \\
\left(P_{11}\right)_{\mathrm{II}}=\frac{2}{R} m_{\eta}\left[\left(M_{1}\right)_{\mathrm{II}}-\left(M_{2}\right)_{\mathrm{II}}\right] a_{2}^{2},
\end{gathered}
$$

and

$$
\frac{d P_{\mathbf{I I}}}{d y}=-\gamma F^{-2}
$$




\section{DIFFERENTIAL SYSTEM GOVERNING STABILITY}

Two-dimensional infinitesimal disturbances are considered in this investigation for simplicity, but this does not imply that Squire's theorem is applicable. Hence, the stability against the three-dimensional disturbances should be studied in a separate investigation.

Let $u_{1}=U+u^{\prime}, u_{2}=V^{\prime}, p=P+p^{\prime}, p_{11}=$ $P_{11}+\sigma_{11}, p_{12}=P_{12}+\sigma_{12}, p_{22}=\sigma_{22}, \epsilon_{11}=\epsilon_{11}^{\prime}, \epsilon_{12}=$ $E_{12}+\epsilon_{12}^{\prime}, \epsilon_{22}=\epsilon_{22}^{\prime}$, in which the strain components are nondimensionalized by the unit $U_{0} / d_{1}$, and $\sigma_{11}$, $\sigma_{12}, \sigma_{22}$, and the quantities denoted by a prime in- dicate the small perturbation from the primary flow. Substituting these quantities into the general equations of motion and the constitutive equations, and neglecting terms of higher orders, we obtain the linearized equations governing the perturbation flow. The linearized equations of motion are

$$
\begin{gathered}
\frac{\partial u^{\prime}}{\partial \tau}+U \frac{\partial u^{\prime}}{\partial x}+v^{\prime} \frac{\partial U}{\partial y}=-\frac{\partial p^{\prime}}{\partial x}+\frac{\partial \sigma_{11}}{\partial x}+\frac{\partial \sigma_{21}}{\partial y} \\
\frac{\partial v^{\prime}}{\partial \tau}+U \frac{\partial v^{\prime}}{\partial x}=-\frac{\partial p^{\prime}}{\partial y}+\frac{\partial \sigma_{21}}{\partial x}+\frac{\partial \sigma_{22}}{\partial y}
\end{gathered}
$$

and the linearized equations of state are

$$
\begin{aligned}
& \sigma_{11}+M_{1}\left[\frac{\partial \sigma_{11}}{\partial \tau}+\frac{\partial \sigma_{11}}{\partial x} U+v^{\prime} \frac{\partial P_{11}}{\partial y}-2\left(\frac{\partial u^{\prime}}{\partial x} P_{11}+\frac{\partial u^{\prime}}{\partial y} P_{12}+\sigma_{12} \frac{d U}{d y}\right)\right]=\frac{2}{R} \epsilon_{11}^{\prime}+\frac{2 M_{2}}{R}\left[\frac{\partial \epsilon_{11}^{\prime}}{\partial \tau}+\frac{\partial \epsilon_{11}^{\prime}}{\partial x} U-\left(\frac{\partial u^{\prime}}{\partial y}+2 \epsilon_{12}^{\prime}\right) \frac{d U}{d y}\right] \\
& \sigma_{12}+M_{1}\left(\frac{\partial \sigma_{12}}{\partial \tau}+\frac{\partial \sigma_{12}}{\partial x} U+v^{\prime} \frac{\partial P_{12}}{\partial y}-\sigma_{22} \frac{d U}{d y}-\frac{\partial v^{\prime}}{\partial x} P_{11}\right) \\
&=\frac{2}{R} \epsilon_{12}^{\prime}+\frac{2 M_{2}}{R}\left(\frac{\partial \epsilon_{12}^{\prime}}{\partial \tau}+\frac{\partial \epsilon_{12}^{\prime}}{\partial x} U+\frac{v^{\prime}}{2} \frac{d^{2} U}{d y^{2}}-\epsilon_{22}^{\prime} \frac{d U}{d y}\right), \\
& \sigma_{22}+M_{1}\left(\frac{\partial \sigma_{12}}{\partial \tau}+\frac{\partial \sigma_{22}}{\partial x} U-2 \frac{\partial v^{\prime}}{\partial x} P_{12}\right)=\frac{2}{R} \epsilon_{22}^{\prime}+\frac{2 M_{2}}{R}\left(\frac{\partial \epsilon_{22}^{\prime}}{\partial \tau}+\frac{\partial \epsilon_{22}^{\prime}}{\partial x} U-\frac{\partial v^{\prime}}{\partial x} \frac{d U}{d y}\right)
\end{aligned}
$$

In addition to these, we have the equation of continuity

$$
\frac{\partial u^{\prime}}{\partial x}+\frac{\partial v^{\prime}}{\partial y}=0
$$

In order to retain the meaning of $R$ and nondimensionalize the pressure by the same unit $\rho_{\mathrm{I}} U_{0}^{2}$ for both layers of liquid, a factor $1 / \gamma$ will arise on the righthand sides of Eq. (13) and another factor $m_{\eta}$ will arise in Eqs. (14) for those terms which contain the Reynolds number $R$, when the lower layer is considered.

Equation (15) permits the use of a stream function $\psi$, in terms of which

$$
u^{\prime}=\frac{\partial \psi}{\partial y}, \quad v^{\prime}=-\frac{\partial \psi}{\partial x} .
$$

As is customary in stability analyses, we assume, that all perturbation quantities contain an exponential time factor, and study the behavior of a spatially periodic disturbance. Thus,

$$
\begin{aligned}
\left(\psi, p^{\prime}, \sigma_{11},\right. & \left.\sigma_{12}, \sigma_{22}\right) \\
= & {\left[\phi(y), f(y), F_{1}(y), F_{2}(y), F_{3}(y)\right] } \\
& \cdot \exp [i \alpha(x-c \tau)],
\end{aligned}
$$

in which $c$ is the eigenvalue sought. The stability or instability is determined by the sign of the imaginary part of $c$, which can be written as $c_{r}+i c_{i}$. The flow is stable when $c_{i}$ is negative and unstable when it is positive. It is understood that more general disturbances can be decomposed into Fourier components, each of which has the form assumed in Eq. (17).

Substituting Eqs. (17) and (16) into Eqs. (13) we eliminate $p^{\prime}$ and obtain, for the two layers in turn,

$$
\begin{aligned}
& i \alpha\left[\left(U_{\mathrm{I}}-c\right)\left(\phi_{\mathrm{I}}^{\prime \prime}-\alpha^{2} \phi_{\mathrm{I}}\right)\right] \\
& \quad=i \alpha\left(F_{1}\right)_{\mathrm{I}}^{\prime}+\left(F_{2}\right)_{\mathrm{I}}^{\prime \prime}+\alpha^{2}\left(F_{2}\right)_{\mathrm{I}}-i \alpha\left(F_{3}\right)_{\mathrm{I}}^{\prime}, \\
& i \alpha \gamma\left[\left(U_{\mathrm{II}}-c\right)\left(\phi_{\mathrm{II}}^{\prime \prime}-\alpha^{2} \phi_{\mathrm{II}}\right)\right] \\
& \quad=i \alpha\left(F_{1}\right)_{\mathrm{II}}^{\prime}+\left(F_{2}\right)_{\mathrm{II}}^{\prime \prime}+\alpha^{2}\left(F_{2}\right)_{\mathrm{II}}-i \alpha\left(F_{3}\right)_{\mathrm{II}}^{\prime}
\end{aligned}
$$

Similarly, substituting Eq. (17) into Eqs. (14) for the two layers in turn, one obtains the linearized equations of state

$$
\begin{aligned}
\left(F_{1}\right)_{i}\left[1+i \alpha\left(M_{1}\right)_{i}\left(U_{i}-c\right)\right] & =\left(M_{1}\right)_{i}\left\{i \alpha\left(P_{11}\right)_{i}^{\prime} \phi_{i}+2\left[i \alpha\left(P_{11}\right)_{i} \phi_{i}^{\prime}+\left(P_{12}\right)_{i} \phi_{i}^{\prime \prime}\right.\right. \\
+ & \left.\left.\left(F_{2}\right)_{i} U_{i}^{\prime}\right]\right\}+i \alpha \beta_{i} \frac{2}{R} \phi_{i}^{\prime}-\beta_{i} \frac{2\left(M_{2}\right)_{i}}{R} \\
& \cdot\left[\alpha^{2} \phi_{i}^{\prime}\left(U_{i}-c\right)+U_{i}^{\prime}\left(2 \phi_{i}^{\prime \prime}+\alpha^{2} \phi_{i}\right)\right]
\end{aligned}
$$




$$
\begin{aligned}
&\left(F_{2}\right)_{i} {\left[1+i \alpha\left(M_{1}\right)_{i}\left(U_{i}-c\right)\right] } \\
&=\left(M_{1}\right)_{i}\left[i \alpha\left(P_{12}\right)_{i}^{\prime} \phi_{i}+\left(F_{3}\right)_{i} U_{i}^{\prime}+\alpha^{2} \phi_{i}\left(P_{11}\right)_{i}\right] \\
&+ \beta_{i} \frac{1}{R}\left(\phi_{i}^{\prime \prime}+\alpha^{2} \phi_{i}\right)+i \alpha \beta_{i} \frac{\left(M_{2}\right)_{i}}{R} \\
& \cdot\left[\left(\phi_{i}^{\prime \prime}+\alpha^{2} \phi_{i}\right)\left(U_{i}-c\right)-\phi_{i} U_{i}^{\prime \prime}+2 \phi_{i}^{\prime} U_{i}^{\prime}\right] \\
&\left(F_{3}\right)_{i}\left[1+i \alpha\left(M_{1}\right)_{i}\left(U_{i}-c\right)\right] \\
&=2\left(M_{1}\right)_{i}\left(P_{11}\right)_{i} \alpha^{2} \phi_{i}-i \alpha \beta_{i} \frac{2}{R} \phi_{i}^{\prime} \\
&+\beta_{i} \frac{2\left(M_{2}\right)_{i}}{R} \alpha^{2}\left[\phi_{i}^{\prime}\left(U_{i}-c\right)-U_{i}^{\prime} \phi_{i}\right],
\end{aligned}
$$

in which the subscript $i$ is taken to be $I$ and II as before for the upper layer and the lower layer of fluids, respectively.

On the boundaries, the zero normal velocity and nonslip condition demand that

$$
\begin{aligned}
\phi_{\mathrm{I}}(1) & =0, & \phi_{\mathrm{I}}^{\prime}(1) & =0, \\
\phi_{\mathrm{II}}(-n) & =0, & \phi_{\mathrm{II}}^{\prime}(-n) & =0 .
\end{aligned}
$$

The continuity of $v^{\prime}$ at the interface demands

$$
\phi_{\mathrm{I}}(0)=\phi_{\mathrm{II}}(0) \text {. }
$$

The kinematic boundary condition at the interface is

$$
\left(\frac{\partial}{\partial \tau}+U_{\mathrm{I}} \frac{\partial}{\partial x}\right) \eta=v^{\prime}=-i \alpha \phi_{\mathrm{I}}(0) \exp [i \alpha(x-c \tau)] \text {. }
$$

From this we find

$$
\eta=\frac{\phi_{\mathrm{I}}(0)}{c^{\prime}} \exp [i \alpha(x-c \tau)],
$$

in which $\eta$ is the deviation of interface from its mean position, and $c^{\prime}=c-U_{\mathrm{I}}(0)$ (or $c^{\prime}=c-b$ ). The continuity in $u^{\prime}$ at interface then demands

$\phi_{\mathrm{I}}^{\prime}(0)+\frac{\phi_{\mathrm{I}}(0)}{c^{\prime}} U_{\mathrm{I}}^{\prime}(0)=\phi_{\mathrm{II}}^{\prime}(0)+\frac{\phi_{\mathrm{II}}(0)}{c^{\prime}} U_{\mathrm{II}}^{\prime}(0)$.

The continuity of shear stress at interface is expressed by $\left[\sigma_{12}+\left(d P_{12} / d y\right)_{\eta}\right]_{\mathrm{I}}=\left[\sigma_{12}+\left(d P_{12} / d y\right) \eta\right]_{\mathrm{II}}$, evaluated at $y=0$. Since $d P_{12} / d y=0$ from Eq. (7c), this boundary condition is simply

$$
\left(F_{2}\right)_{\mathrm{I}}=\left(F_{2}\right)_{\mathrm{II}} \text { at } y=0 .
$$

The continuity of normal stress at interface is expressed by

$$
\begin{aligned}
\left(-\boldsymbol{p}^{\prime}-\frac{d P}{d y} \eta\right. & \left.+\sigma_{22}\right)_{\mathrm{I}} \\
& -\left(-\boldsymbol{p}^{\prime}-\frac{d P}{d y} \eta+\sigma_{22}\right)_{\mathrm{II}}=-S \frac{\partial^{2} \eta}{\partial x^{2}}
\end{aligned}
$$

in which $S=T / \rho_{\mathrm{I}} U_{0}^{2} d_{1}$, and $T$ is the surface tension, and again, variables are evaluated at $y=0$. We utilize the first equation in (13) to evaluate $f$ and hence $p^{\prime}$ for either liquid. With the results so obtained, and with $\eta$ evaluated from Eq. (21), and $d P_{1} / d y$ and $d P_{11} / d y$ from Eq. (12), the normal stress condition can be rewritten as

$$
\begin{aligned}
-\alpha\left(c^{\prime} \phi_{\mathrm{I}}^{\prime}+a_{1} \phi_{\mathrm{I}}\right)-\alpha\left(F_{1 / \mathrm{I}}+i\left(F_{2}\right)_{\mathrm{I}}^{\prime}\right. \\
+\alpha\left(F_{3}\right)_{\mathrm{I}}+\alpha \gamma\left(c^{\prime} \phi_{\mathrm{II}}^{\prime}+a_{2} \phi_{\mathrm{II}}\right) \\
+\alpha\left(F_{1}\right)_{\mathrm{II}}-i\left(F_{2}\right)_{\mathrm{II}}^{\prime}-\alpha\left(F_{3}\right)_{\mathrm{II}} \\
=\alpha\left[(\gamma-1) F^{-2}+\alpha^{2} S\right] \frac{\phi_{\mathrm{I}}(0)}{c^{\prime}}
\end{aligned}
$$

evaluated at $y=0$.

The differential system governing the stability consists of Eqs. (18), (19), and (20a-h).

\section{SOLUTION OF THE DIFFERENTIAL SYSTEM}

The regular perturbation technique is used to solve the eigenvalue problem for long waves, or for $\alpha \ll 1$. From Eqs. (19) we obtain as $\mathrm{Lai}^{5} \mathrm{did}$,

$$
\begin{aligned}
\left(F_{1}\right)_{\mathrm{I}}= & \frac{4 \Delta M}{R} U_{\mathrm{I}}^{\prime} \phi_{\mathrm{I}}^{\prime \prime}+O(\alpha), \\
\left(F_{2}\right)_{\mathrm{I}}= & \frac{1}{R} \phi_{\mathrm{I}}^{\prime \prime}+i \alpha \frac{\Delta M}{R}\left[U_{\mathrm{I}}^{\prime \prime} \phi_{\mathrm{I}}-2 \phi_{\mathrm{I}}^{\prime} U_{\mathrm{I}}^{\prime}\right. \\
& \left.-\phi_{\mathrm{I}}^{\prime \prime}\left(U_{\mathrm{I}}-c\right)\right]+O\left(\alpha^{2}\right),
\end{aligned}
$$

$\left(F_{3}\right)_{\mathrm{I}}=-i \alpha \frac{2}{R} \phi_{\mathrm{I}}^{\prime}+O\left(\alpha^{2}\right)$,

$\left(F_{1}\right)_{\mathrm{II}}=\frac{4 \Delta M}{R} m_{\eta} m_{\lambda} U_{\mathrm{II}}^{\prime} \phi_{\mathrm{II}}^{\prime \prime}+O(\alpha)$

$\left(F_{2}\right)_{\mathrm{II}}=\frac{1}{R} m_{\eta} \phi_{\mathrm{II}}^{\prime \prime}+i \alpha \frac{1}{R} m_{\eta} m_{\lambda} \Delta M$

$$
\cdot\left[U_{\mathrm{II}}^{\prime \prime} \phi_{\mathrm{II}}-2 \phi_{\mathrm{II}}^{\prime} U_{\mathrm{II}}^{\prime}-\phi_{\mathrm{II}}^{\prime}\left(U_{\mathrm{II}}-c\right)\right]+O\left(\alpha^{2}\right),
$$

$\left(F_{3}\right)_{\mathrm{II}}=-i \alpha \frac{2}{R} m_{\eta} \phi_{\mathrm{II}}^{\prime}+O\left(\alpha^{2}\right)$,

in which

$$
\begin{gathered}
\Delta M=\left(M_{1}\right)_{\mathrm{I}}-\left(M_{2}\right)_{\mathrm{I}}, \\
m_{\mathrm{\lambda}}=\frac{\left(M_{1}\right)_{\mathrm{II}}-\left(M_{2}\right)_{\mathrm{II}}}{\left(M_{1}\right)_{\mathrm{I}}-\left(M_{2}\right)_{\mathrm{I}}}=\frac{\left(\lambda_{1}\right)_{\mathrm{II}}-\left(\lambda_{2}\right)_{\mathrm{II}}}{\left(\lambda_{1}\right)_{\mathrm{I}}-\left(\lambda_{2}\right)_{\mathrm{I}}} .
\end{gathered}
$$

Substituting Eqs. (22) into Eqs. (18) and (20a-h), we have, to the first power of $\alpha$

$$
\begin{aligned}
& \phi_{\mathrm{I}}^{\prime \prime \prime \prime}-i \alpha R\left(U_{\mathrm{I}}-c\right) \phi_{\mathrm{I}}^{\prime \prime}+i \alpha \Delta M\left\{4\left(U_{\mathrm{I}}^{\prime} \phi_{\mathrm{I}}^{\prime \prime}\right)^{\prime}\right. \\
& \left.+\left[U_{\mathrm{I}}^{\prime \prime} \phi_{\mathrm{I}}-2 \phi_{\mathrm{I}}^{\prime} U_{\mathrm{I}}^{\prime}-\phi_{\mathrm{I}}^{\prime \prime}\left(U_{\mathrm{I}}-c\right)\right]^{\prime \prime}\right\}=0
\end{aligned}
$$




$$
\begin{array}{rr}
\phi_{\mathrm{II}}^{\prime \prime \prime}-i \alpha R \frac{\gamma}{m_{\eta}}\left(U_{\mathrm{II}}-c\right) \phi_{\mathrm{II}}^{\prime \prime}+i \alpha \Delta M m_{\lambda}\left\{4\left(U_{\mathrm{II}}^{\prime} \phi_{\mathrm{II}}^{\prime \prime}\right)^{\prime}\right. \\
\left.+\left[U_{\mathrm{II}}^{\prime \prime} \phi_{\mathrm{II}}-2 \phi_{\mathrm{II}}^{\prime} U_{\mathrm{II}}^{\prime}-\phi_{\mathrm{II}}^{\prime \prime}\left(U_{\mathrm{II}}-c\right)\right]^{\prime \prime}\right\}=0 \\
\phi_{\mathrm{I}}(1)=0, \quad \phi_{\mathrm{I}}^{\prime}(1)=0, & (25 \mathrm{a}, \mathrm{b}) \\
\phi_{\mathrm{II}}(-n)=0, \quad \phi_{\mathrm{II}}^{\prime}(-n)=0, & (25 \mathrm{c}, \mathrm{d}) \\
\phi_{\mathrm{I}}(0)=\phi_{\mathrm{II}}(0), & (25 \mathrm{e})
\end{array}
$$

$$
\phi_{I}^{\prime}(0)-\phi_{I I}^{\prime}(0)+\frac{\phi_{I}(0)}{c^{\prime}}\left[U_{\mathrm{I}}^{\prime}(0)-U_{\mathrm{II}}^{\prime}(0)\right]=0
$$

$$
\begin{aligned}
\phi_{\mathrm{I}}^{\prime \prime} & +i \alpha \Delta M\left[U_{\mathrm{I}}^{\prime \prime} \phi_{\mathrm{I}}-2 \phi_{\mathrm{I}}^{\prime} U_{\mathrm{I}}^{\prime}\right. \\
- & \left.\phi_{\mathrm{I}}^{\prime \prime}\left(U_{\mathrm{I}}-c\right)\right]-m_{\eta} \phi_{\mathrm{II}}^{\prime \prime}-i \alpha \Delta M m_{\eta} m_{\lambda} \\
& \cdot\left[U_{\mathrm{II}}^{\prime \prime} \phi_{\mathrm{II}}-2 \phi_{\mathrm{II}}^{\prime} U_{\mathrm{II}}^{\prime}-\phi_{\mathrm{II}}\left(U_{\mathrm{II}}-c\right)\right]=0, \\
\phi_{\mathrm{I}}^{\prime \prime \prime} & +i \alpha R\left(c^{\prime} \phi_{\mathrm{I}}^{\prime}+a_{1} \phi_{\mathrm{I}}\right)+i \alpha \Delta M \\
& \cdot\left\{4 U_{\mathrm{I}}^{\prime} \phi_{\mathrm{I}}^{\prime \prime}+\left[U_{\mathrm{I}}^{\prime \prime} \phi_{\mathrm{I}}-2 \phi_{\mathrm{I}}^{\prime} U_{\mathrm{I}}^{\prime}-\phi_{\mathrm{I}}^{\prime \prime}\left(U_{\mathrm{I}}-c\right)\right]^{\prime}\right\} \\
- & m_{\eta} \phi_{\mathrm{II}}^{\prime \prime \prime}-i \alpha R \gamma\left(c^{\prime} \phi_{\mathrm{II}}^{\prime}+a_{2} \phi_{\mathrm{II}}\right) \\
- & i \alpha \Delta M m_{\eta} m_{\lambda}\left\{4 U_{\mathrm{II}}^{\prime} \phi_{\mathrm{II}}^{\prime \prime}\right. \\
+ & {\left.\left[U_{\mathrm{II}}^{\prime \prime} \phi_{\mathrm{II}}-2 \phi_{\mathrm{II}}^{\prime} U_{\mathrm{II}}^{\prime}-\phi_{\mathrm{II}}^{\prime \prime}\left(U_{\mathrm{II}}-c\right)\right]^{\prime}\right\} } \\
+ & i \alpha R(\gamma-1) F^{-2} \frac{\phi_{\mathrm{I}}(0)}{c^{\prime}}=0,
\end{aligned}
$$

In Eqs. (25g) and (25h), all variables are evaluated at $y=0$, where primes, except the prime on $c$, indicate the derivative with respect to $y$. All terms containing $\alpha^{2}$ and higher orders of $\alpha$ are ignored since this investigation will include only the firstorder approximation. In fact, the method of regular perturbation adopted here can accommodate as high an order of approximation as we desire by including the higher order terms of $\alpha$.

Following the approach of $\mathrm{Yih}^{2}$ by expanding the eigenfunctions and eigenvalue in a power series of wavenumber $\alpha$

$$
\begin{aligned}
\phi_{\mathrm{I}} & =\phi_{0}+\alpha \phi_{1}+\alpha^{2} \phi_{2}+\cdots, \\
\phi_{\mathrm{II}} & =\chi_{0}+\alpha \chi_{1}+\alpha^{2} \chi_{2}+\cdots, \\
c & =c_{0}+\alpha c_{1}+\alpha^{2} c_{2}+\cdots .
\end{aligned}
$$

\section{A. The Zeroth-Order Approximation}

The zeroth approximation gives us the governing equations

$$
\begin{aligned}
& \phi_{0}^{\prime \prime \prime \prime}=0, \\
& \chi_{0}^{\prime \prime \prime \prime}=0 ;
\end{aligned}
$$

and the boundary conditions

\footnotetext{
C.-S. Yih, Phys. Fluids 6, 321 (1963).
}

$$
\begin{aligned}
\phi_{0}(1)=0, & \phi_{0}^{\prime}(1)=0, \\
\chi_{0}(-n)=0, \quad \chi_{0}^{\prime}(-n) & =0, \\
\phi_{0}(0)-\chi_{0}(0) & =0, \\
\phi_{0}^{\prime}(0)-\chi_{0}^{\prime}(0)+\frac{\phi_{0}(0)}{c_{0}^{\prime}}\left(a_{1}-a_{2}\right) & =0, \\
\phi_{0}^{\prime \prime}(0)-m_{\eta} \chi_{0}^{\prime \prime}(0) & =0, \\
\phi_{0}^{\prime \prime \prime}(0)-m_{\eta} \chi_{0}^{\prime \prime \prime}(0) & =0 .
\end{aligned}
$$

Solution of the differential system is found to be

$$
\begin{aligned}
& \phi_{0}=1+A_{1} y+A_{2} y^{2}+A_{3} y^{3}, \\
& \chi_{0}=1+B_{1} y+B_{2} y^{2}+B_{3} y^{3},
\end{aligned}
$$

in which

$$
\begin{aligned}
A_{1} & =-\frac{m_{\eta}+3 n^{2}+4 n^{3}}{2 n^{2}(1+n)}, & B_{1} & =\frac{n^{3}+m_{\eta}(4+3 n)}{2 m_{\eta} n(1+n)}, \\
B_{2} & =\frac{n^{3}+m_{\eta}}{n^{2} m_{\eta}(1+n)}, & B_{3} & =\frac{n^{2}-m_{\eta}}{2 n^{2} m_{\eta}(1+n)}, \\
A_{2} & =m_{\eta} B_{2}, & A_{3} & =m_{\eta} B_{3} .
\end{aligned}
$$

The eigenvalue $c_{0}$ is determined by

$$
\begin{aligned}
c_{0}^{\prime}=c_{0}-b & =-\frac{a_{1}-a_{2}}{A_{1}-B_{1}} \\
& =\frac{2 n^{2} m_{\eta}(1+n)\left(a_{1}-a_{2}\right)}{m_{\eta}^{2}+2 n m_{\eta}\left(2+3 n+2 n^{2}\right)+n^{4}} .
\end{aligned}
$$

The solution of the zeroth-order approximation is the same as that given by $\mathrm{Yih}^{\mathbf{8}}$ for Newtonian fluids.

\section{B. The First-Order Approximation}

Having obtained the eigenvalue $c_{0}$ and eigenfunctions $\phi_{0}$ and $\chi_{0}$, we put them into the equations governing the first-order approximation and obtain

$$
\begin{aligned}
\phi_{1}^{\prime \prime \prime \prime}= & i R\left[6 a_{1} A_{3} y^{2}\right. \\
& \left.+2\left(a_{1} A_{2}-3 A_{3} c_{0}^{\prime}\right) y-2 c_{0}^{\prime} A_{2}\right], \\
\chi_{1}^{\prime \prime \prime \prime}= & i R \frac{\gamma}{m_{\eta}}\left[6 a_{2} B_{2} y^{2}\right.
\end{aligned}
$$

$$
\left.+2\left(a_{2} B_{2}-3 B_{3} c_{0}^{\prime}\right) y-2 c_{0}^{\prime} B_{2}\right] .
$$

The general solutions of Eq. (29) can be written as $\phi_{1}=\Delta A_{1} y+\Delta A_{2} y^{2}+\Delta A_{3} y^{3}+i R H_{1}(y)$,

$\chi_{1}=\Delta B_{1} y+\Delta B_{2} y^{2}+\Delta B_{3} y^{3}+i R \frac{\gamma}{m_{\eta}} H_{2}(y)$,

in which

$$
H_{1}(y)=\frac{a_{1} A_{3}}{60} y^{6}+\frac{a_{1} A_{2}-3 A_{3} c_{0}^{\prime}}{60} y^{5}-\frac{c_{0}^{\prime} A_{2}}{12} y^{4},
$$


and

$$
H_{2}(y)=\frac{a_{2} B_{3}}{60} y^{6}+\frac{a_{2} B_{2}-3 B_{3} c_{0}^{\prime}}{60} y^{5}-\frac{c_{0}^{\prime} B_{2}}{12} y^{4} .
$$

The six constants of integration and hence the eigenvalue will be determined from the boundary conditions. The boundary condition (22h), in the firstorder approximation, can be simplified by using the relations in the zero-order approximation, and the simplified form is

$$
\begin{aligned}
\phi_{1}^{\prime \prime \prime}(0)- & m_{\eta} \chi_{1}^{\prime \prime \prime}(0) \\
& +i R\left[(\gamma-1)\left(\frac{1}{c_{0}^{\prime} F^{2}}-c_{0}^{\prime} A_{1}-a_{1}\right)\right] \\
& +2 i \Delta M\left[a_{1} A_{2}+3 c_{0}^{\prime} A_{3}\right. \\
& \left.-m_{\eta} m_{\lambda}\left(a_{2} B_{2}+3 c_{0}^{\prime} B_{2}\right)\right]=0 .
\end{aligned}
$$

The first-order approximation of the boundary condition (25e) is automatically satisfied by (30) because we take the terms of zero power in $y$ for $\phi_{1}$ and $\chi_{1}$ to be zero by following Yih's ${ }^{9}$ argument. The first-order approximations of the boundary conditions can be derived straightforwardly from Eqs. (25). Applying the boundary conditions on $\phi_{1}$ and $\chi_{1}$, we obtain

$$
\begin{aligned}
\Delta A_{1}+\Delta A_{2}+\Delta A_{3}+i R H_{1}(1) & =0 \\
\Delta A_{1}+2 \Delta A_{2}+3 \Delta A_{3}+i R H_{1}^{\prime}(1) & =0, \\
n \Delta B_{1}-n^{2} \Delta B_{2}+n^{3} \Delta B_{3}-i R \gamma \frac{1}{m_{\eta}} H_{2}(-n) & =0 \\
\Delta B_{1}-2 n \Delta B_{2}+3 n^{2} \Delta B_{3}+i R \gamma \frac{1}{m_{\eta}} H_{2}^{\prime}(-n) & =0, \\
\Delta A_{1}-\Delta B_{1}+\frac{a_{2}-a_{1}}{\left(c_{0}^{\prime}\right)^{2}} c_{1} & =0, \\
\Delta A_{2}-m_{\eta} \Delta B_{2}+i \Delta M K_{1} & =0, \\
\Delta A_{3}-m_{\eta} \Delta B_{3}+i R K_{2}+i \Delta M K_{3} & =0,
\end{aligned}
$$

in which

$$
\begin{aligned}
& K_{1}=a_{1} A_{1}-c_{0}^{\prime} A_{2}-m_{\eta} m_{\lambda}\left(a_{2} B_{1}-c_{0}^{\prime} B_{2}\right), \\
& K_{2}=\frac{1}{6}(\gamma-1)\left(\frac{1}{c_{0}^{\prime} F^{2}} c_{0}^{\prime} A_{1}-a_{1}\right),
\end{aligned}
$$

and

$$
K_{3}=\frac{1}{3}\left[a_{1} A_{2}+3 c_{0}^{\prime} A_{3}-m_{\eta} m_{\lambda}\left(a_{2} B_{2}+3 c_{0}^{\prime} B_{3}\right)\right] .
$$

We note that the non-Newtonian effect is felt only through the conditions of continuity of shear and normal stresses at the interface. Separating the eigenvalue $c_{1}$ into real and imaginary as $c_{1}=\left(c_{1}\right)_{r}+$ $i\left(c_{1}\right)_{i}$, we obtain from Eqs. (32) the results $\left(c_{1}\right)_{r}=0$ and

$\left(c_{1}\right)_{i}=R J_{1}\left(\gamma, n, m_{\eta}\right)+\Delta M J_{2}\left(n, m_{\eta}, m_{\lambda}\right)$,

in which

$$
\begin{aligned}
J_{1}= & \frac{\left(c_{0}^{\prime}\right)^{2}}{m_{\eta}\left(a_{1}-a_{2}\right)}\left[m_{\eta}\left[H_{1}^{\prime}(1)-2 H_{1}(1)\right]\right. \\
& -\frac{2}{n} \gamma H_{2}(-n)-\gamma H_{2}^{\prime}(-n)-n^{2} K_{2} \\
& +\frac{m_{\eta}-n^{2}}{2(1+n)}\left(H_{1}(1)-H_{1}^{\prime}(1)-\frac{\gamma}{n^{2}} H_{2}(-n)\right. \\
& \left.\left.-\frac{\gamma}{n} H_{2}^{\prime}(-n)-2 n K_{2}\right)\right]
\end{aligned}
$$

and

$$
J_{2}=\frac{\left(c_{0}^{\prime}\right)^{2}}{m_{\eta}\left(a_{1}-a_{2}\right)}\left(\frac{n^{2}-m_{\eta}}{2(1+n)} K_{1}-\frac{n\left(n+m_{\eta}\right)}{1+n} K_{3}\right)
$$

\section{RESULTS AND DISCUSSION}

The $\left(c_{1}\right)_{i}$ given by Eq. (33) is the criterion of stability. It consists of two parts: $R J_{1}$ due to the viscosity and $\Delta M J_{2}$ due to the elasticity of the liquids. For $\Delta M=0$, Eq. (33) becomes

$$
\left(c_{1}\right)_{i}=R J_{1}\left(\gamma, n, m_{\eta}\right),
$$

which reproduces Yih's ${ }^{8}$ result for Newtonian fluids.

As pointed out by $Y$ ih, we can verify directly from Eq. (34) that

$$
\frac{m_{\eta}}{n^{2}} J_{1}\left(n, m_{\eta}\right)=J_{1}\left(\frac{1}{n}, \frac{1}{m_{\eta}}\right)
$$

when $\gamma=1$. This is still true in this investigation. In addition, one from Eq. (35) can also verify that

$$
\frac{1}{m_{\lambda}} J_{2}\left(n, m_{\eta}, m_{\lambda}\right)=J_{2}\left(\frac{1}{n}, \frac{1}{m_{\eta}}, \frac{1}{m_{\lambda}}\right),
$$

which is to say that the part of $\left(c_{1}\right)_{i}$ due to the elasticity also remains the same when the two superposed layers of fluid are interchanged, as it should from a physical point of view. The factor $1 / m_{\lambda}$ on the left-hand side of Eq. (36) arose as long as the definition of $\Delta M$ is retained before and after the interchange.

To see whether the elasticity of the liquids has any stabilizing influence, numerical calculations have been carried out. The results are illustrated by curves shown in Figs. 2-4. Since $\lambda_{1}>\lambda_{2}, \Delta M$ is positive. 
Keeping Eq. (33) in mind, we see that the elasticity in liquid causes the flow stability if $J_{2}<0$ and instability if $J_{2}>0$.

For $n<0.5$, the elasticity in liquid stabilizes the flow if $m_{\eta}<1$, no matter what values $m_{\mathrm{\lambda}}$ may have, and destabilizes the flow if $m_{\eta}>1$. The stabilizing effect is increased with increasing $m_{\lambda}$, but the value of $m_{\mathrm{\lambda}}$ seems to have only a slight influence on the growth rate of the disturbance for cases in which the elasticity destabilizes the flow. The variation of $J_{2}$ with $m_{\mathrm{\lambda}}$ and $m_{\eta}$ for the special case, $n=0.1$, is shown in Fig. 2.

For $n$ increasing from 0.5 toward 1 ; the range of value of $m_{\eta}$ for which the elasticity of the fluids stabilizes the flow becomes narrower for $m_{\eta}<1$, and wider for $m_{\eta}>1$. Figure 3 illustrates the variation of $J_{2}$ with $m_{\lambda}$ and $m_{\eta}$ for the special case, $n=1$. Whether stabilizing or destabilizing, the damping rate or growth rate of the disturbance attributable to the action of elasticity of the liquids varies only slightly with $m_{\lambda}$ when $m_{\lambda}<1$, but greatly with $m_{\lambda}$ when $m_{\lambda}>1$.

The values of $J_{2}$ for $n>1$ can be calculated from the values of $J_{2}$ for $n<1$ which are plotted in figures by using the correlation (36).

Figure 4 shows the variation of $J_{2}$ with $m_{\lambda}$ and $n$ for $m_{\eta}=0.4$. From this we see that nonuniformity in elastic property of the liquids stabilizes the flow when $n<1$, and destabilizes the flow when $n \geq 1$ for this particular value of $m_{\eta}$.

As one can see from Figs. 2-4, $J_{2}$ vanishes whenever $m_{\eta}$ approaches $1 . J_{2}$ is other than zero when $m_{\eta} \neq 1$. This result reveals that the stabilizing or destabilizing effect of the elasticity in liquid is absent in the absence of viscosity stratification, and is only brought about when the viscosity varies.

Numerical calculation also shows that the values of $J_{1}$ and $J_{2}$ can be of opposite sign for the case $\gamma=1$ with the depth ratio $n$ larger than about $0.5 . J_{1}$ can be positive while $J_{2}$ is negative or vice versa depending on the values of $n, m_{\eta}$, and $m_{\lambda}$. This indicates that the effect of the elasticity in the liquid can be either stabilizing or destabilizing. Computations for $J_{1}$ are also carried out for the cases of $\gamma=1.1,1.2$, and 1.4 each for four different values of the Froude number $F=0.1,0.5,1$, and 2 . The results demonstrate that $J_{1}$ decreases with increasing the value of $\gamma$. This feature is expected since the effect of gravity is to stabilize horizontal flows when there is a negative density gradient in the direction of the vertical. For the cases of $F=0.1(\gamma \geq 1.1)$, and $F=0.5(\gamma \geq 1.2)$, the destabilizing effect of viscosity variation in Newtonian fluid is completely overshadowed by the

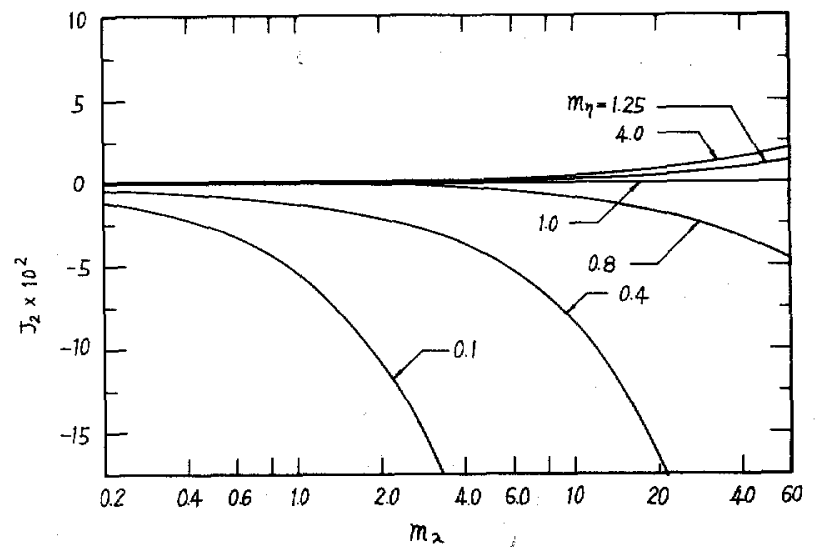

FIG. 2. Variation of $J_{2}$ with the elasticity ratio $m_{\lambda}$ for various values of the viscosity ratio $m_{\eta}$ for the case of depth ratio $n=0.1$.

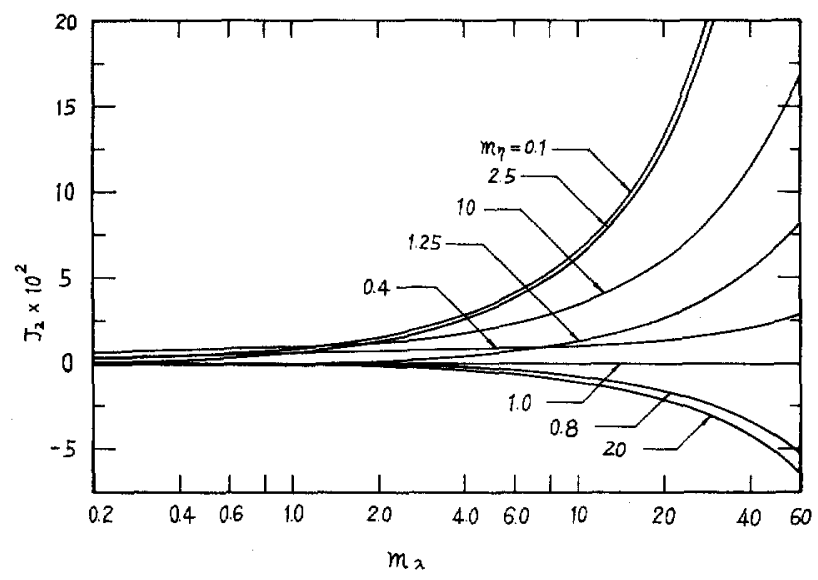

FIG. 3. Variation of $J_{2}$ with the elasticity ratio $m_{\lambda}$ for various values of the viscosity ratio $m_{\eta}$ for the case of depth ratio $n=1$.

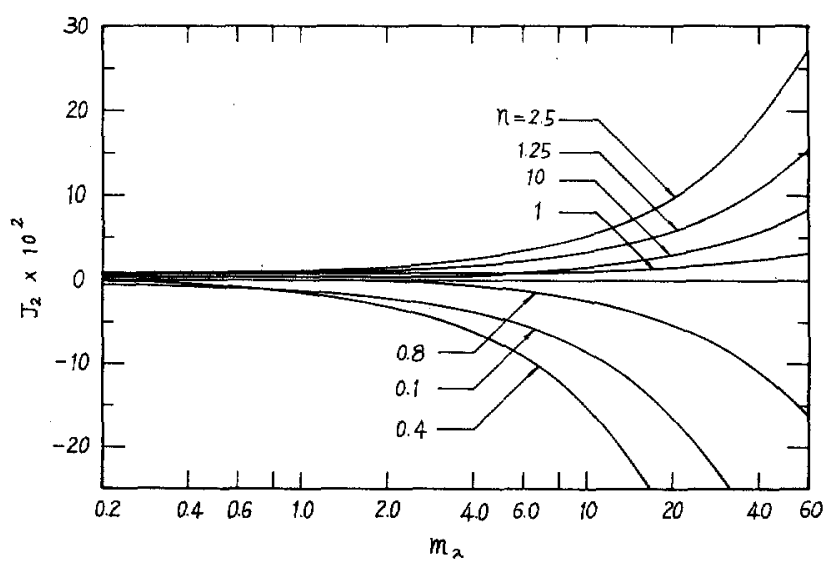

FIG. 4. Variation of $J_{2}$ with the elasticity ratio $m_{\lambda}$ for various values of the depth ratio, $n$, for the case of viscosity ratio $m_{\eta}=0.4$. 
stabilizing effect of gravity. In these cases, the positive value of $J_{2}$ indicates that the elasticity of the liquid destabilizes the flow while the negative value of $J_{2}$ indicates that the elasticity behavior in liquid increases the degree of stability of the flow. For $F \geq 1$, the destabilizing effect of viscosity variation in Newtonian fluid is not completely overshadowed by the stabilizing effect of gravity, at least for $\gamma \leq 1.4$. The oppositeness in sign of $J_{1}$ and $J_{2}$ is still there for certain values of $n, m_{\eta}$, and $m_{\lambda}$. In order to shorten the paper, the numerical results for $J_{1}$ are not presented. For the case of $\gamma=1$, the value of $J_{1}$ can be found in Yih's ${ }^{8}$ paper.
From the foregoing it can be concluded that the elasticity of the liquids destabilizes the flow for certain values of $n, m_{\eta}$, and $m_{\lambda}$, but stabilizes it for other values of these variables. The detailed results are shown in the figures mentioned before.

\section{ACKNOWLEDGMENTS}

The author wishes to express his sincere appreciation to Professor Chia-Shun Yih for his guidance and encouragement during the period of this research.

The financial support from the National Science Foundation and the Army Research Office (Durham) is highly appreciated. 